\title{
BORBOLETAS (LEPIDOPTERA) AMEAÇADAS DE EXTINÇÃO EM MINAS GERAIS, BRASIL ${ }^{1}$
}

\author{
Mirna M. Casagrande ${ }^{2}$ \\ Olaf H.H. Mielke ${ }^{2}$ \\ Keith S. Brown Jr. ${ }^{3}$
}

\begin{abstract}
BUTtERFLIES (LEPIDOPTERA) CONSIDERED AS THREATENED IN MINAS GERAIS, BRAZIL. The twenty species of butterflies (diurnal Lepidoptera) considered as threatened in the Minas Gerais (by statute) are described and discussed in relation to distribution, appearance and known records.

KEY WORDS. Lepidoptera, butterflies threatened, Brazil
\end{abstract}

O presente trabalho visa ilustrar as espécies incluídas na "Lista de espécies ameaçadas de extinção do Estado de Minas Gerais" publicada pela COPAM (Conselho Estadual de Política Ambiental) (Minas Gerais 1996). Detalhes com informações ecológicas serão incluídos no "Livro vermelho das espécies ameaçadas do estado de Minas Gerais" a ser editado pela Fundação Biodiversitas.

As borboletas pertencem à ordem Lepidoptera que compreende aproximadamente 150.000 espécies conhecidas, das quais 19.000 são borboletas (HEPPNER 1991), sendo que no Brasil devem ocorrer ao todo 40.000 espécies, das quais 3.300 espécies de borboletas (BROWN 1996a,b). As borboletas são quase todas diurnas, com algumas poucas exceções (Hesperiidae, Lycaenidae e Nymphalidae: Satyrinae) e se diferenciam das mariposas pelas antenas clavadas e nunca terem um frênulo no ângulo umeral da asa posterior acoplado ao retináculo na face ventral da asa anterior (uma só exceção na Austrália com frênulo - Euschemon rafflesia Macleay, 1827, Hesperiidae). As borboletas são mais bem conhecidas que as mariposas e é possível reconhecer algumas espécies como consideradas ameaçadas de extinção, na maioria dos casos por destruição do seu habitat típico pelo avanço dos sistemas antrópicos que já substituiram mais de $90 \%$ dos sistemas naturais e $95 \%$ da Floresta Atlântica no estado de Minas Gerais. Tais destruições quase sempre eliminam boa parte das plantas utilizadas como alimento pelas lagartas das borboletas, ou mesmo as flores ou a vegetação preferidas pelos adultos, levando certas espécies de distribuição geográfica muito restrita, rara ou muito especializadas, a desaparecerem.

1) Contribuição número 1050 do Departamento de Zoologia, Universidade Federal do Paraná.

2) Departamento de Zoologia, Universidade Federal do Paraná. Caixa Postal 19020, 81531-990 Curitiba, Paraná, Brasil. Bolsista do CNPq.

3) Departamento de Zoologia, Instituto de Biologia, Universidade Estadual de Campinas. Caixa postal 6109, 13083-970 Campinas, São Paulo, Brasil. 
Vinte destas borboletas, entre as mais raras das mais de 1.600 espécies conhecidas em Minas Gerais, foram incluídas nesta lista, de forma a abranger os principais sistemas de vegetação e climas (biomas) existentes no estado. São sete espécies de Floresta Atlântica perenefólia ou semidecidual de planície e terras onduladas (principalmente nos vales médios dos Rios Doce, Mucuri, Muriaé e Pomba) - Arawacus aethesa, Hypoleria fallens, Heliconius nattereri, Moschoneura methymna, Dasyophthalma vertebralis, Hyalyris fiammetta e Hyalyris leptalina, sete de matas mais frias em altitudes maiores (também no bioma da Floresta Atlântica) - Charonias theano, Ortilia polinella, Callicore hydarnis, Dasyophthalma geraensis, Prepona deiphile, Tithorea harmonia caissara e Cyanophrys bertha, duas de campos rupestres (refúgios de grandes altitudes) - Orobrassolis ornamentalis e Nirodia belphegor, e quatro do bioma dos Cerrados (sendo duas de matas ciliares - Parides burchellanus e Parides panthonus jaguarae, uma de matas de cabeceira - Agrias claudina godmani, e uma de cerrado arbustivo - Magnastigma julia). Três destas espécies não são vistas há mais de 50 anos nas localidades outrora conhecidas - Parides panthonus jaguarae, Hyalyris fiammetta e Dasyophthalma vertebralis. Se não forem encontradas em outros lugares nos próximos anos poderiam ser consideradas extintas em Minas Gerais (todas eram conhecidas também de estados vizinhos onde existem habitats típicos preservados, ainda não inventariados).

De fato há poucos estudos da fauna de Lepidoptera de Minas Gerais; os mais completos são listas publicadas por HAMBLETON \& FORBES (1935; mariposas), BROWN \& MiELKE (1967a,b; 1968; borboletas da região dos cerrados e Belo Horizonte), ZIKÁN \& ZIKÁN (1968, borboletas da região do Itatiaia), EBERT (1969; borboletas de Poços de Caldas) e BRown (1972a; borboletas de diversos lugares). Muitos estudos ainda são necessários para elucidar os aspectos ecológicos das espécies mencionadas a seguir (FORTUNATO \& RUSZCZYK 1997).

Catorze espécies foram, após examinação criteriosa dos dados e discussão, foram excluídas desta lista, ou de outras listas de espécies ameaçadas (BERNARDES et. al. 1990; BRown 1991, 1993, 1996a,b; CASAgRANDE \& Mielke 1993, 1995) por uma de duas razões: (1) não são seguramente reconhecidas em Minas Gerais Heraclides himeros himeros (Hopffer, 1865) - Papilionidae, Papilioninae; Hesperocharis emeris (Boisduval, 1836) (= catasticta Röber, 1909), Pyrrhybris flava Oberthür, 1896 - Pieridae, Pierinae; Melinaea mnasias thera C. Felder \& R. Felder, 1865 - Nymphalidae, Ithomiinae e Doxocopa zalmunna (Butler, 1869) - Nymphalidae, Apaturinae; ou (2) as populações em Minas Gerais são vigorosas e protegidas - Cunizza hirlanda planasia Fruhstorfer, 1910 - Pieridae, Pierinae; Doxocopa laurona (Schaus, 1902) - Nymphalidae, Apaturinae; Actinote morio Oberthür, 1917, Actinote quadra Schaus, 1902 - Nymphalidae, Heliconiinae; Arcas ducalis (Westwood, 1851) - Lycaenidae, Theclinae; Alesa prema (Godart, 1824), Eucorna sanarita Schaus, 1902, Synargis ethelinda (Hewitson, 1870, Mesenopsis albivitta (Lathy, 1904) - Riodinidae, Riodininae. Em contrapartida, diversas espécies não contempladas na "Lista Oficial do IBAMA" ou de seu "Anexo" (espécies em estudo) (BERNARDES et.al. 1990) foram incluídas na lista do estado de Minas Gerais por serem de interesse especial para a proteção nesta unidade da federação (Cyanophrys 
bertha, Magnastigma julia, Ortilia polinella). Inevitavelmente esta lista estadual necessitará de uma revisão, provavelmente para acrescentar espécies a medida que os estudos lepidopterológicos se tornarem mais consistentes, e o estudo das espécies mais raras, ou restritas, em seu habitat seja feito.

\section{ESPÉCIES INCLUÍDAS NA LISTA}

\section{Parides burchellanus (Westwood, 1872) \\ Papilioninae, Papilionidae}

Fig. 1

Situação no Brasil definida pelo IBAMA (Portaria 1522/89): não consta da portaria.

Situação em Minas Gerais (deliberação COPAM 041-95): ameaçada, em perigo. Distribuição em Minas Gerais: transição entre os biomas Cerrado e Floresta Atlântica. Carmo do Rio Claro, Matozinhos (Fazenda Jaguara), região de Uberaba (Farinha Podre, localidade do tipo).

Ocorrência em outros Estados: Distrito Federal (Brasília), Goiás e São Paulo.

Parides burchellanus é uma borboleta grande (asa anterior $45 \mathrm{~mm}$ ) de asas negras com cinco a seis manchas pequenas, submarginais, ovaladas e vermelhas na asa posterior; escova androconial do macho na margem interna da asa posterior de um pardo acinzentado e franjas da margem externa da asa posterior brancas.

\section{Parides panthonus jaguarae (Foetterle, 1902) \\ Papilioninae, Papilionidae}

Fig. 2

Situação no Brasil definida pelo IBAMA (Portaria 1522/89): não consta da portaria.

Situação em Minas Gerais (deliberação COPAM 041-95): ameaçada, criticamente em perigo.

Distribuição em Minas Gerais: transição entre os biomas Floresta Atlântica e Cerrado, a norte de Belo Horizonte. Matozinhos (Fazenda Jaguara, março: FOETTERLE 1902).

Ocorrência em outros Estados: São Paulo (Batatais, Casabranca).

Parides panthonus jaguarae é uma borboleta grande (asa anterior $45 \mathrm{~mm}$ ) de asas negras e com cinco a sete manchas pequenas, submarginais, romboidais e vermelhas na asa posterior; escova androconial do macho na margem interna da asa posterior de um pardo acinzentado e franjas da margem externa da asa posterior rosas. A subespécie é muito semelhante à Parides burchellanus, anteriormente mencionada, da qual se diferencia pela cor das franjas, entre as veias, e pela forma dos pontos vermelhos normalmente mais romboidais em jaguarae (TYLER et al. 1994), enquanto são mais ovalados em burchellanus; caráter mais facilmente observável na face ventral da asa. 

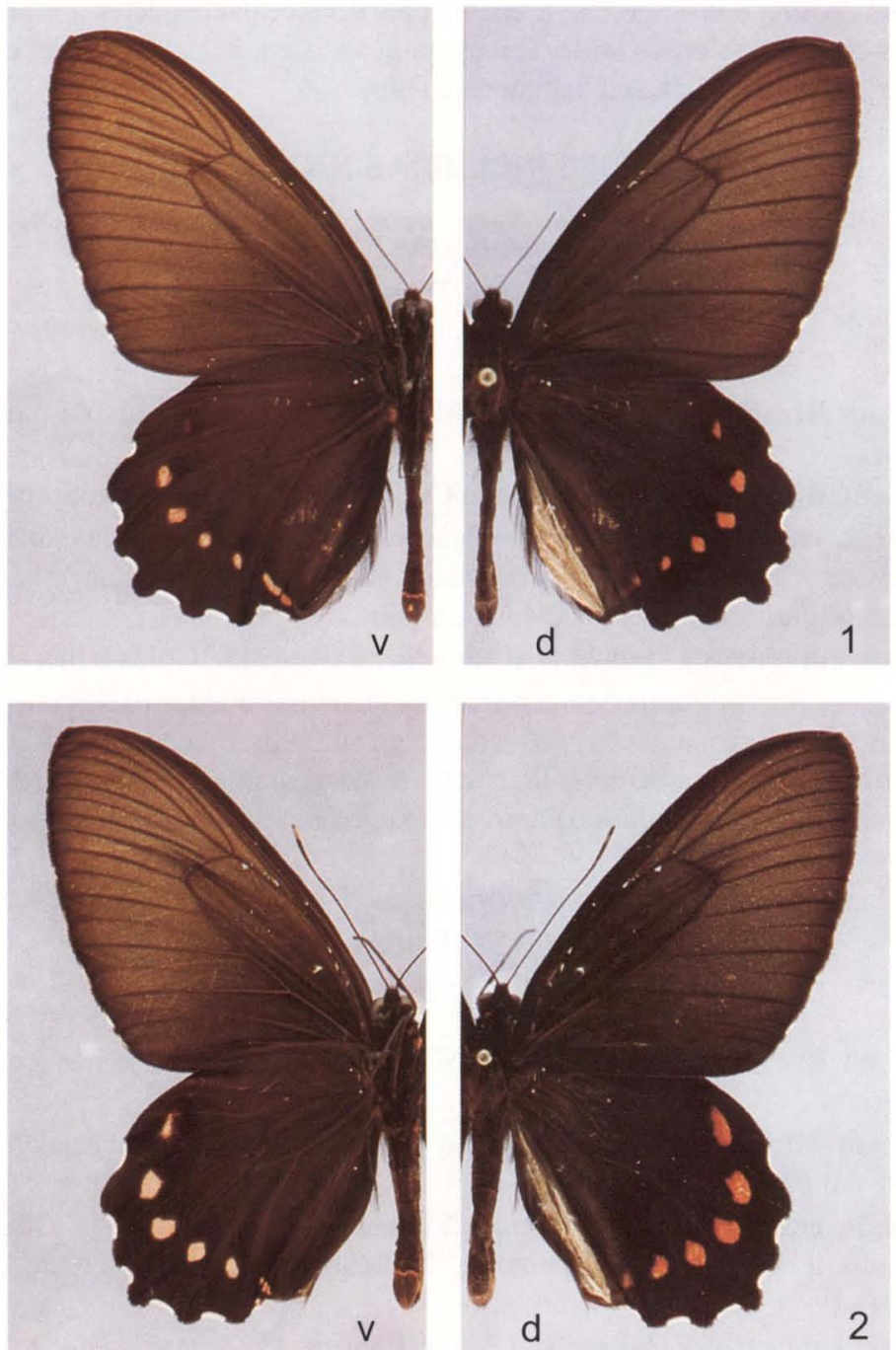

Figs 1-2. (1) Parides burchellanus; (2) Parides panthonus jaguarae. Machos dorsal (d) e ventral (v). 


\section{Charonias theano (Boisduval, 1836) \\ Pierinae, Pieridae}

Figs 3,4

Situação no Brasil definida pelo IBAMA (Portaria 1522/89): não consta da portaria.

Situação em Minas Gerais (deliberação COPAM 041-95): ameaçada, criticamente em perigo.

Distribuição em Minas Gerais: mata estacional semidecidual de altitude da Floresta Atlântica. Poços de Caldas (EBERT 1969), Cambuquira, Passa Quatro (ZIKÁN \& ZIKÁN 1968), Caxambu e Conçeição dos Ouros.

Ocorrência em outros Estados: São Paulo (Amparo, Guarulhos - Serra da Cantareira, Avaré), Paraná (Candido de Abreu, Ponta Grossa) e Santa Catarina (Joinville) (CASAGRANDE \& MiELKE 1993, 1995).

Charonias theano é uma borboleta de tamanho médio (asa anterior $27 \mathrm{~mm}$ no macho e $30 \mathrm{~mm}$ na fêmea) e de asas alongadas. O dimorfismo sexual é acentuado, sendo que o macho possui as asas negras, na face dorsal da asa anterior com manchas brancas, semidifusas, no limite distal da célula discal, no centro da asa, acima e abaixo da veia $2 \mathrm{~A}$ e três ou quatro manchas apicais; na face dorsal da asa posterior com manchas semelhantes na célula discal, na base do espaço entre as veias M1-M2 e M2-M3 e na área anal; na face ventral da asa anterior com as mesmas manchas, porém amarelo-claras e maiores, incluindo mais uma linear na célula discal e outra na margem externa entre as veias $\mathrm{CuA} 1-\mathrm{CuA} 2$; na face ventral da asa posterior quase totalmente amarelo-clara, salvo as veias negras e manchas brancas submarginais. A fêmea possue nas suas faces dorsal e ventral todas as manchas como no macho, mas de um amarelo ocráceo e um pouco maiores em ambas as faces, salvo as da margem externa da face ventral da asa posterior brancas.

\section{Moschoneura methymna (Godart, 1819) \\ Dismorphiinae, Pieridae}

Fig. 5

Situação no Brasil definida pelo IBAMA (Portaria 1522/89: ameaçada de extinção.

Situação em Minas Gerais (deliberação COPAM 041-95): ameaçada, vulnerável.

Distribuição em Minas Gerais: matas densas e úmidas ao longo dos riachos de serra na Floresta Atlântica. Raul Soares.

Ocorrência em outros Estados: Bahia (Camacã), Espírito Santo (Santa Teresa), Rio de Janeiro (Petrópolis, Teresópolis, Parque Nacional da Serra dos Órgãos, Vila Inhomirim, Xerém, Itatiaia - Parque Nacional), Santa Catarina (CASAGRANDE \& MIELKE 1993, 1995)

Moschoneura methymna é uma borboleta de tamanho médio com as asas alongadas (asa anterior $25 \mathrm{~mm}$ ). O macho e a fềmea são semelhantes. Face dorsal 

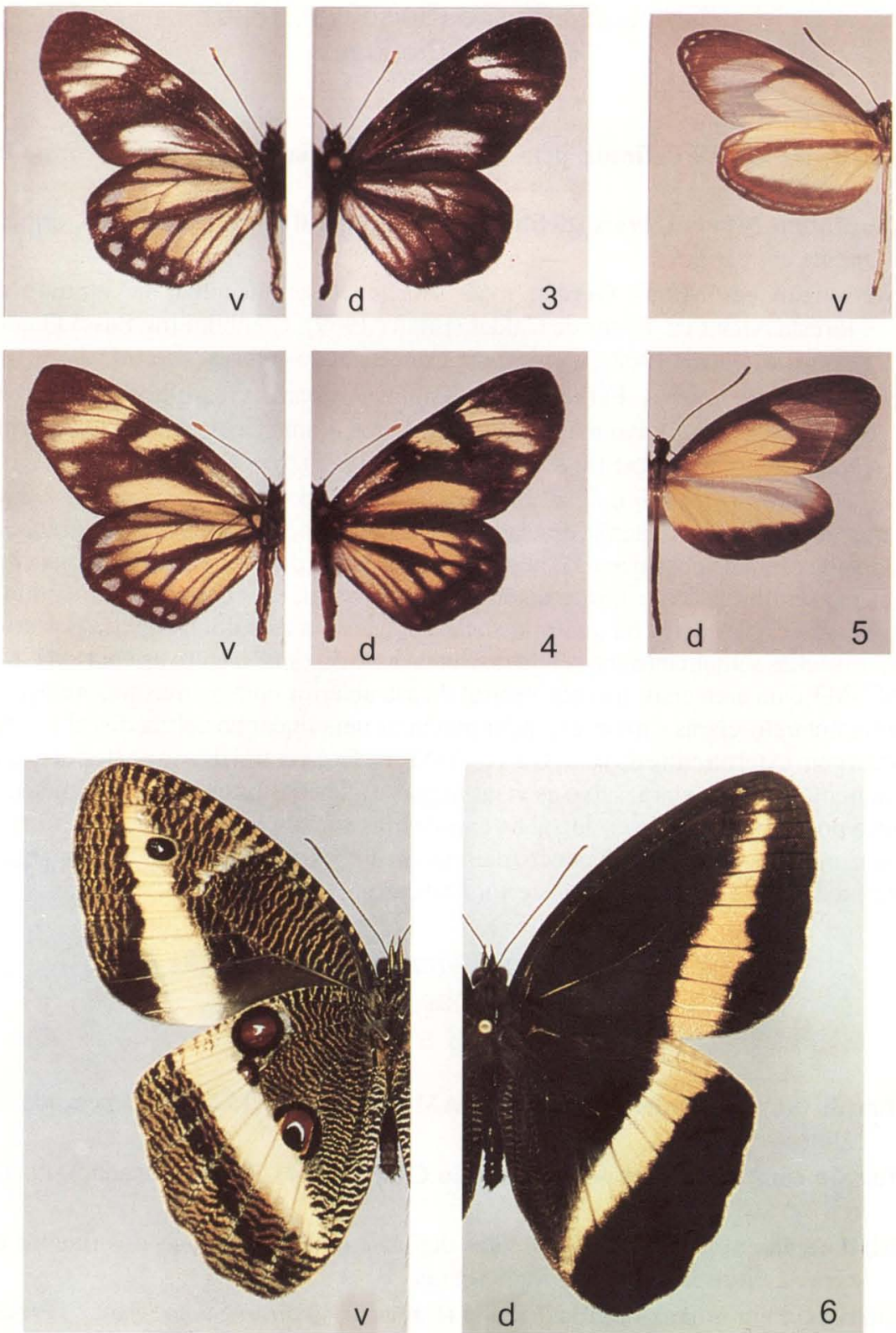

Figs 3-6. (3) Charonias theano, macho; (4) fêmea; (5) Moschoneura methymna, macho; (6) Dasyophthalma geraensis, macho. Dorsal (d) e ventral (v). 
da asa anterior com a área basal amarelo-clara e as áreas costal e distal negras, esta com larga mancha subapical acinzentada; na asa posterior igualmente amarela, sendo as áreas costal esbranquiçada e a marginal externa negra; face ventral semelhante, porém o ápice da asa anterior mais acinzentado e o torno branco, na asa posterior a margem costal amarelo-clara separada por faixa negra da área central e o negro da margem externa inclue uma faixa alaranjada.

\section{Dasyophthalma geraensis Rebel, 1922 \\ Brassolinae, Nymphalidae}

Fig. 6

Situação no Brasil definida pelo IBAMA (Portaria 1522/89): não consta da portaria.

Situação em Minas Gerais (deliberação COPAM 014-95): ameaçada, vulnerável.

Distribuição em Minas Gerais: em matas de altitude na Floresta Atlântica. Delfim Moreira, Itamonte, Passa Quatro e Virgínia.

Ocorrência em outros estados: Rio de Janeiro (Itatiaia - Parque Nacional), São Paulo (Piquete).

Dasyophthalma geraensis é uma borboleta grande (asa anterior $45 \mathrm{~mm}$ ) de asas marrom escuro, na face dorsal atravessada por faixa oblíqua de uma amarelo intenso e com azul metálico na base entre o cúbito e a margem interna; ventralmente a faixa amarela mais clara e todo o restante com matizes em castanho médio sobre amarelo claro, na asa anterior com um ocelo negro de pupila branca entre as veias M1-M2 e na asa posterior com dois ocelos, um alaranjado escuro com pupila branca entre e sobre Sc+R1-Ml e outro com pupila negra, marginada de branco no lado proximal em M3-CuA1. A fêmea difere do macho por possuir uma mancha amarela subapical isolada na face dorsal da asa anterior em R3-R4.

\section{Dasyophthalma vertebralis Butler, 1869 \\ Brassolinae, Nymphalidae}

Figs 7,8

Situação no Brasil definida pelo IBAMA (Portaria 1522/89): provavelmente extinta.

Situação em Minas Gerais (deliberação COPAM 041-95): ameaçada, provavelmente extinta.

Distribuição em Minas Gerais: Alto Rio Mucuri.

Ocorrência em outros Estados: Espírito Santo (Alegre, Muqui, Santa Leopoldina). A ocorrência "Pará", mencionada na descrição original (BUTLER 1869), deve ser um engano.

Dasyophthalma vertebralis é uma borboleta grande (asa anterior $50 \mathrm{~mm}$ ) de asas negras com as margens externas, uma faixa da base ao ápice na asa anterior (contornando a célula discal) e outra faixa semelhante mais larga entre o ângulo anal 

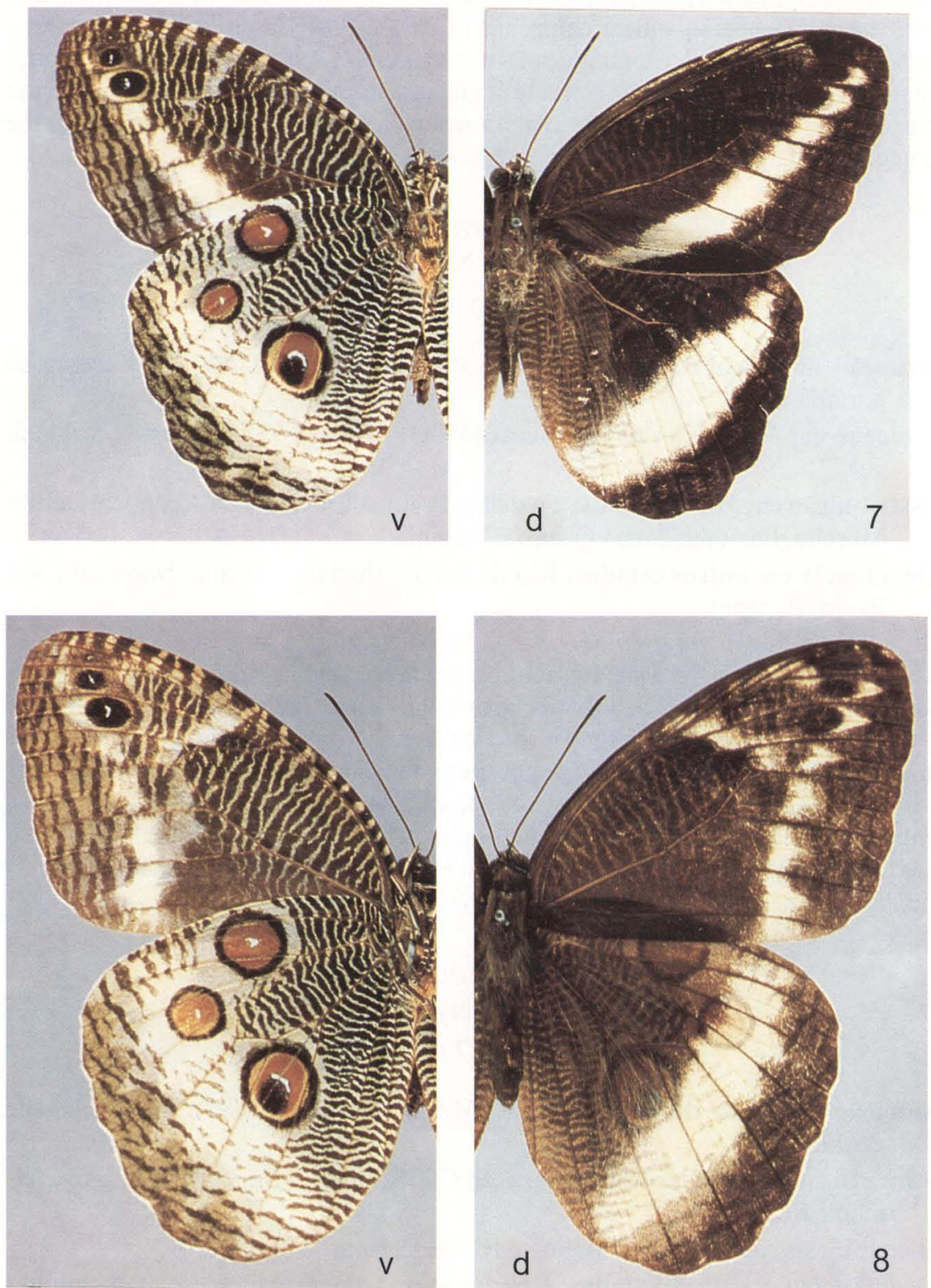

Figs 7-8. (7) Dasyophthalma vertebralis, macho; (8) fêmea. Dorsal (d) e ventral (v). 
e o ápice da asa posterior de um amarelo claro. A fêmea tem na asa anterior manchas amarelas na base das medianas, no meio das células cubitais, e entre as radiais e medianas (subapicais). Ventralmente é esbranquiçada com matizes em marron escuro, na asa anterior com dois ocelos negros de pupilas brancas próximos ao ápice e na asa posterior com três ocelos alaranjados, dois com pupila branca e o mais inferior, com pupila negra.

\section{Orobrassolis ornamentalis (Stichel, 1906) Brassolinae, Nymphalidae}

Fig. 9

Situação no Brasil definida pelo IBAMA (Portaria 1522/89): ameaçada de extinção.

Situação em Minas Gerais: ameaçada, em perigo.

Distribuição em Minas Gerais: nos campos de altitude da Floresta Atlântica. Piranguçu.

Ocorrência em outros Estados: São Paulo (Campos do Jordão - Parque Estadual), Paraná (Castro) (CASAGRANDE \& MielKe 1993, 1995).

Orobrassolis ornamentalis é uma borboleta de tamanho médio (asa anterior $33 \mathrm{~mm}$ ) de coloração amarelo parda, com fina linha submarginal, negra e irregular, com pequeno ocelo preto apical; ventralmente mais clara e com muitas linhas finas pretas. Asa posterior com dois ocelos entre Sc+Rs-M1 e CuA1-CuA2.

\section{Agrias claudina godmani Fruhstorfer, 1895 Charaxinae, Nymphalidae}

Fig. 10

Situação no Brasil definida pelo IBAMA (Portaria 1522/89): não consta da portaria.

Situação em Minas Gerais (deliberação COPAM 041-95): ameaçada, vulnerável.

Distribuição em Minas Gerais: florestas de cabeceira ou espigões na transição entre os biomas Cerrado e Floresta Atlântica. Belo Horizonte, Paracatu, Uberlândia.

Ocorrência em outros Estados: Distrito Federal (Brasília), Goiás, Mato Grosso (Diamantino, Chapada dos Guimarães, Alto Garças, Barra do Bugres), São Paulo (Cajuru).

Agrias claudina godmani é uma borboleta grande (asa anterior $45-50 \mathrm{~mm}$ ) e uma das mais cobiçadas pelos colecionadores, junto com as outras espécies e subespécies deste gênero, sendo chamadas de um modo genérico "Rainha das borboletas" (KESSELRING 1989). As asas são anegradas com reflexos azuis e grandes manchas vermelhas na face dorsal, enquanto que na face ventral da asa posterior há um padrão complexo de linhas, manchas pretas e anéis submarginais azuis sobre um fundo negro, o que leva as espécies a serem consideradas como as borboletas " 88 das grandes". 

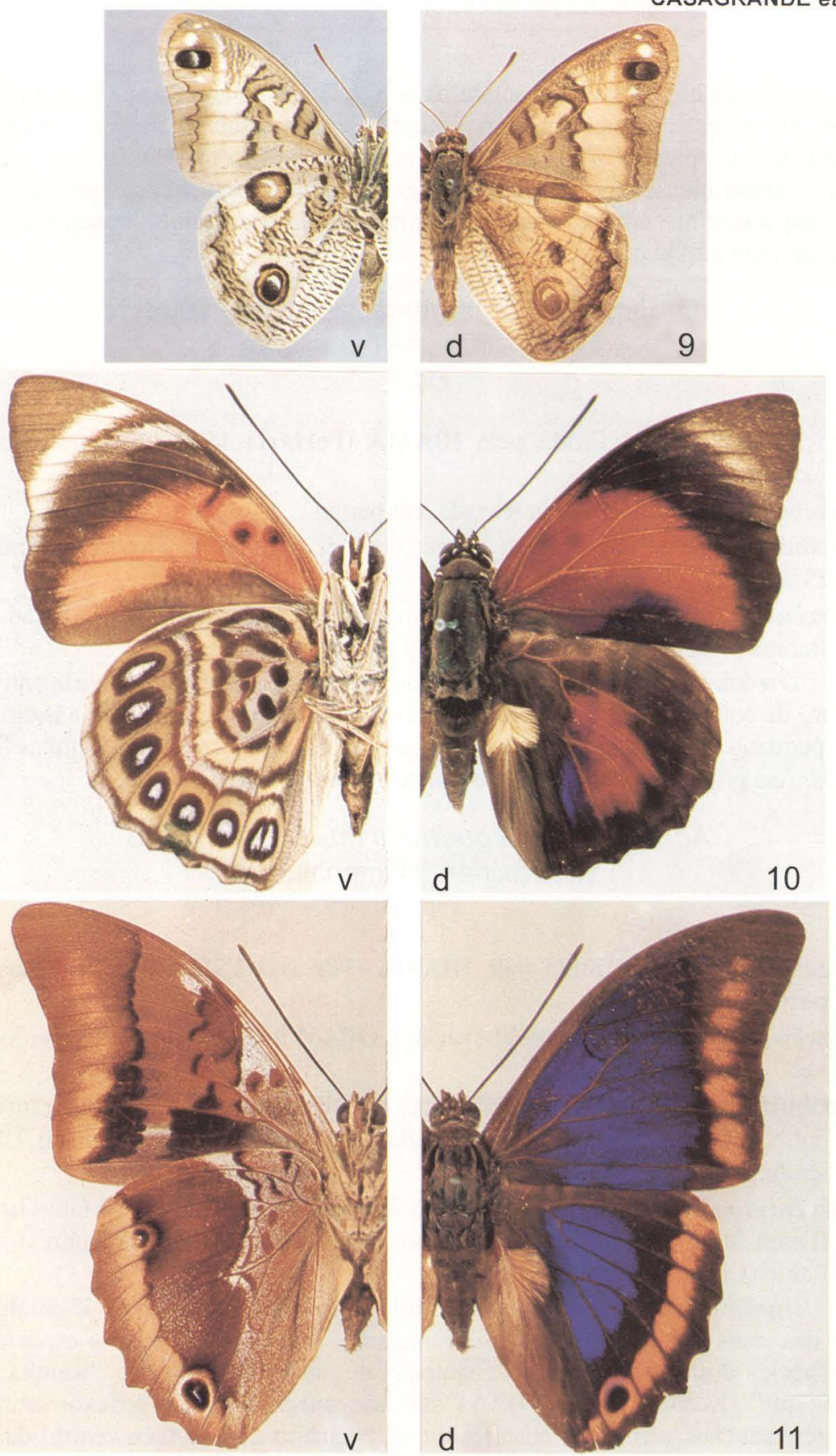

Figs 9-11. (9) Orobrassolis ornamentalis, macho; (10) Agrias claudina godmani, macho; (11) Prepona deiphile, macho. Dorsal (d) e ventral (v). 


\section{Prepona deiphile (Godart, [1824]) \\ Charaxinae, Nymphalidae}

Fig. 11

Situação no Brasil definida pelo IBAMA (Portaria 1522/89): não consta da portaria.

Situação em Minas Gerais (deliberação COPAM 041-95): ameaçada, vulnerável.

Distribuição em Minas Gerais: serras úmidas da Floresta Atlântica. Caparaó, Vale Verde.

Ocorrência em outros Estados: Espírito Santo (Santa Teresa), Rio de Janeiro (Cachoeiras de Macacu, Itatiaia - Parque Nacional, Petrópolis), São Paulo (Jundiaí, Alto da Serra),Paraná (Guaratuba, Castelhanos), Santa Catarina (São Bento do Sul).(CASAgRande \& Mielke 1993, 1995).

Prepona deiphile é uma borboleta grande (asa anterior $50-60 \mathrm{~mm}$ ) de asa pontuda e reflexos azuis-violetas acentuados nos dois terços basais das asas, limitados por uma fileira de manchas submarginais de um amarelo ouro na face dorsal. O lado ventral das asas é marrom avermelhado com pontos, linhas, manchas, ocelos negros e desenhos estreitos brancos na parte central. Não há outra espécie com a qual possa ser confundida no Brasil; há espécies próximas nos Andes e nas montanhas do México.

\section{Callicore hydarnis (Godart, [1824]) \\ Limenitidinae, Nymphalidae}

Fig. 12

Situação no Brasil definida pelo IBAMA (Portaria 1522/89): não consta da portaria.

Situação em Minas Gerais (deliberação COPAM 041-95): ameaçada, em perigo. Distribuição em Minas Gerais: serra altas no extremo sudeste do estado. Itamonte, Passa Quatro (BRown 1991, 1996a, b).

Ocorrência em outros Estados: Rio de Janeiro (Itatiaia - Parque Nacional, Teresópolis, Parque Nacional da Serra dos Órgãos).

Callicore hydarnis é uma borboleta de tamanho médio (asa anterior $20 \mathrm{~mm}$ ). A face dorsal das asas é de azul brilhante e com uma faixa mediana nas anteriores, vermelha; a face ventral da asa posterior apresenta desenhos semelhantes aos das espécies de Diaethria Billberg, 1820 (as borboletas "88"), no entanto, com as pupilas brancas e não negras.

\section{Heliconius nattereri C. Felder \& R. Felder, 1865 Heliconiinae, Nymphalidae}

Figs 13,14

Situação no Brasil definida pelo IBAMA (Portaria 1522/89): ameaçada de extinção.

Situação em Minas Gerais (deliberação COPAM 041-95): ameaçada, em perigo. 

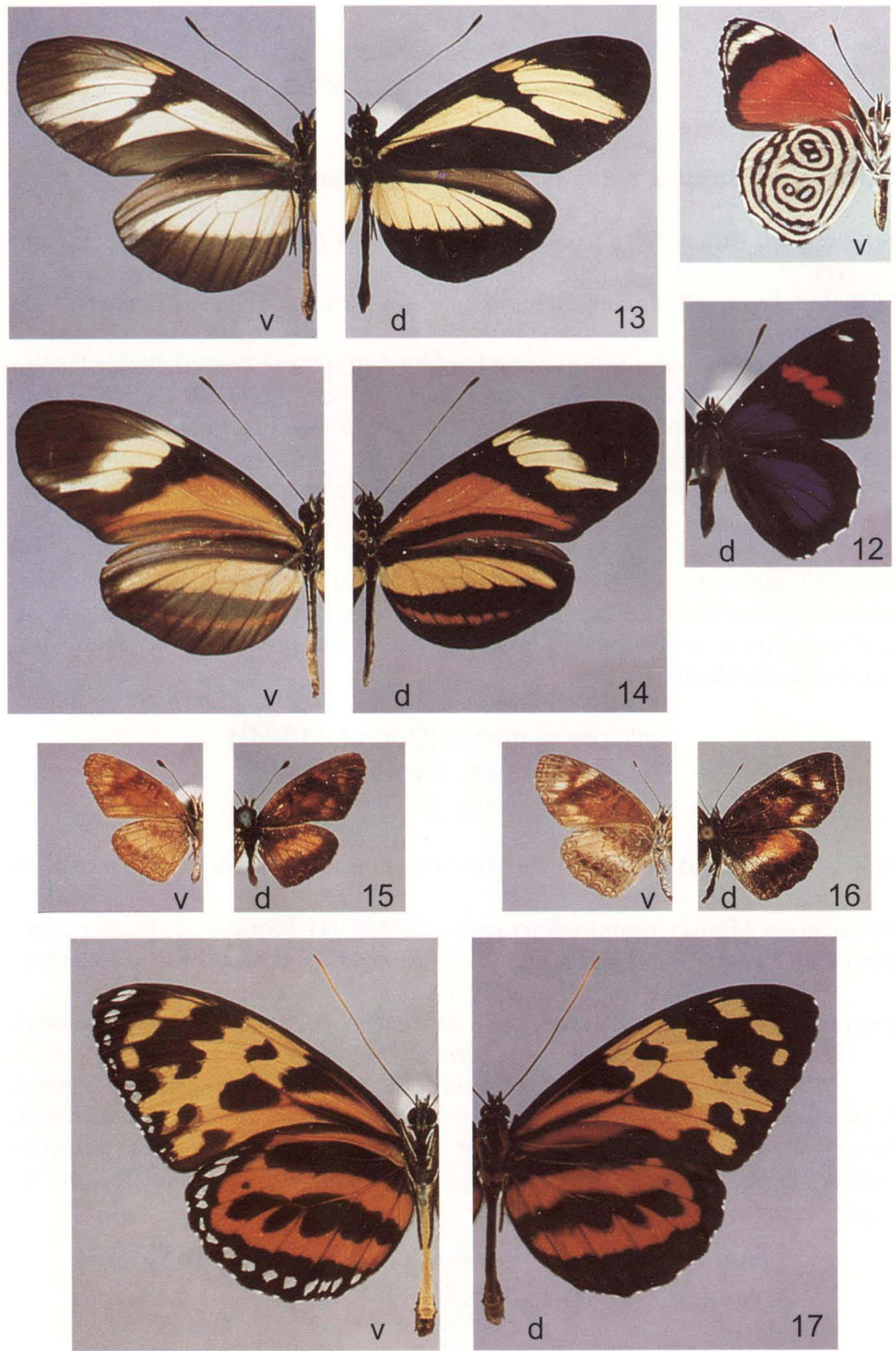

Figs 12-17. (12) Callicore hydarnis, macho; (13) Heliconius nattereri, macho; (14) fêmea; (15) Ortilia polinella, macho; (16) fêmea; (17) Tithorea harmonia caissara, macho. Dorsal (d) e ventral ( $v)$. 
Distribuição em Minas Gerais: matas de encosta da Floresta Atlântica no Vale do Rio Doce e na região de Caratinga. Marliéria. Caratinga.

Ocorrência em outros Estados: Bahia (Camacã, Amargosa), Espírito Santo (Santa Leopoldina, Santa Teresa, Santa Maria), Rio de Janeiro (Casimiro de Abreu).

Heliconius nattereri é uma borboleta de tamanho médio-grande (asa anterior $35-40 \mathrm{~mm}$ ) de asas muito alongadas. O macho possue as asas negras com duas faixas amarelas na asa anterior, uma longitudinal central, outra subapical, e outra longitudinal na asa posterior. A fêmea possui a faixa longitudinal central da asa anterior e uma faixa submarginal adicional na asa posterior alaranjadas, esta podendo invadir a faixa longitudinal (BRown 1970, 1972b). A face ventral é semelhante, porém o negro é substituido por acinzentado.

Ortilia polinella (Hall, 1928)

Nymphalinae, Nymphalidae

Figs 15, 16

Situação no Brasil definida pelo IBAMA (Portaria 1522/89): não consta da portaria.

Situação em Minas Gerais (deliberação COMPAM 041-95): ameaçada, em perigo.

Distribuição em Minas Gerais: mata estacional semidecidual da Floresta Atlântica. Teófilo Otoni (Vale do Rio São Jacinto) (HigGINS 1981).

Ocorrência em outros Estados: Espírito Santo, Rio de Janeiro (São Fidelis, Itatiaia - Parque Nacional), São Paulo (Piquete).

Ortilia polinella é uma borboleta pequena (asa anterior $15 \mathrm{~mm}$ ) e constitui, juntamente com Ortilia dicoma (Hewitson, 1864), um grupo de espécies com as asas anteriores alongadas. A face dorsal das asas é de um castanho escuro com poucas manchas alaranjadas difusas e na asa posterior com faixa difusa larga e lúnulas submarginais da mesma cor. A face ventral de um castanho alaranjado com um desenho indistinto de linhas finas. A fêmea possui manchas amarelo-alaranjadas maiores e a faixa da asa posterior é esbranquiçada e muito estreita na metade basal; o desenho da face ventral é mais definido que no macho.

\section{Tithorea harmonia caissara (Zikán, 1941) Ithomiinae, Nymphalidae}

Fig. 17

Situação no Brasil definida pelo IBAMA (Portaria 1522/89): não consta da portaria.

Situação em Minas Gerais (deliberação COPAM 041-95): ameaçada, em perigo.

Distribuição em Minas Gerais: serras de altitude média com solos ricos e matas íngremes ao longo de córregos, nos extremos leste e sul da Floresta Atlântica. Conceição dos Ouros e Pouso Alegre.

Ocorrência em outros Estados: Espírito Santo (Alegre), Rio de Janeiro (Itatiaia Parque Nacional), São Paulo (Jundiaí, Serra Negra e Cantareira). 

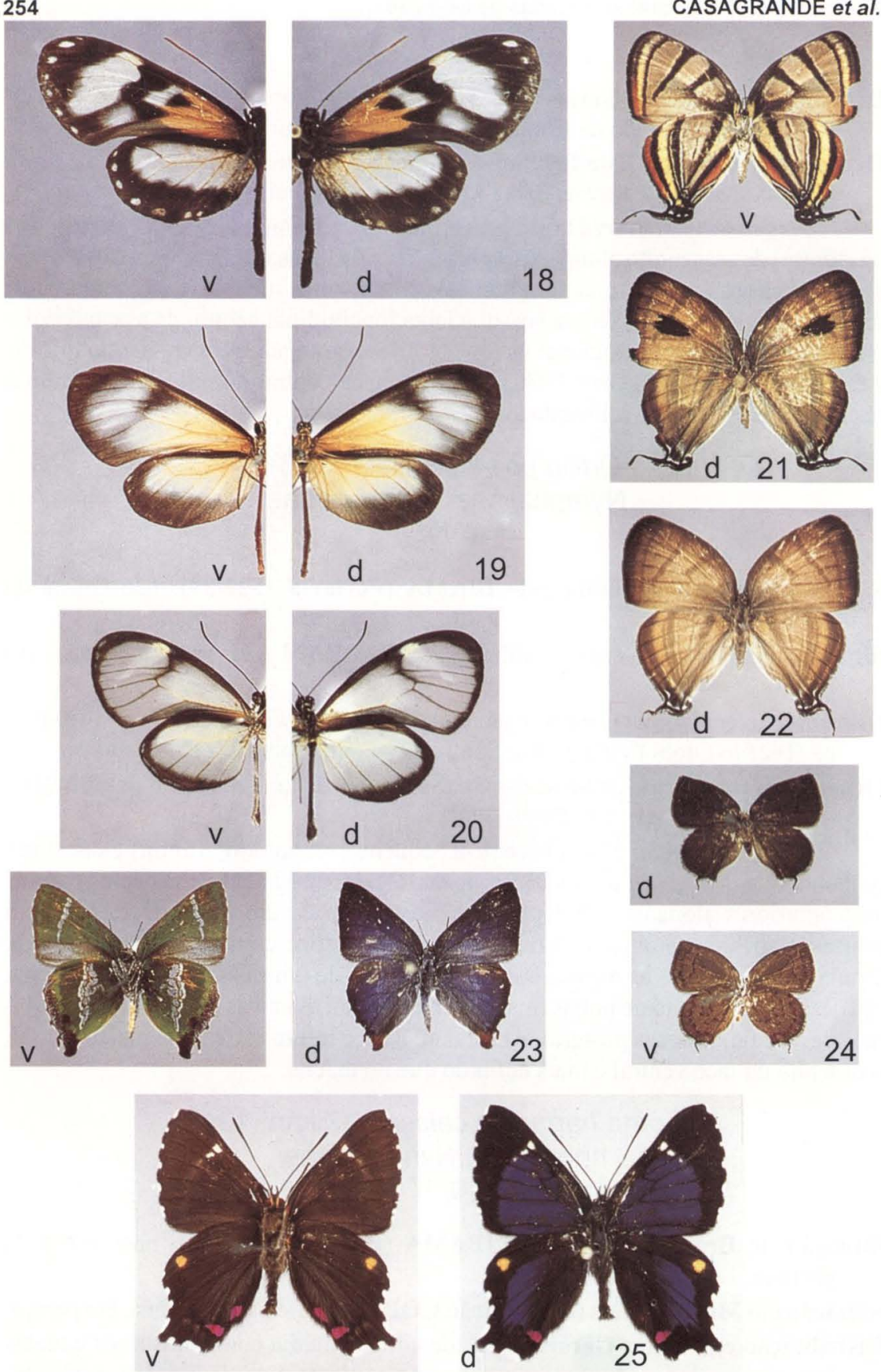

Figs 18-25. (18) Hyalyris fiammetta, macho; (19) Hyalyris leptalina, macho; (20) Hypoleria fallens, macho; (21) Arawacus aethesa, macho; (22) fêmea; (23) Cyanophrys bertha, macho; (24) Magnastigma julia, macho; (25) Nirodia belphegor, macho. Dorsal (d) e ventral (v). 
Tithorea harmonia caissara é uma borboleta grande (asa anterior $37-40 \mathrm{~mm}$ ) de asa anterior alongada, mas não tanto como na maioria dos Ithomiinae, com um padrão "tigre" muito escuro, chocolate, com listras e bordas pretas, e uma faixa larga subapical amarelo-limão na asa anterior. Assim assemelha-se a Heliconius ethilla narcaea, forma "satis" Weymer, 1875 (Heliconiinae) e à forma escura de Lycorea halia discreta Haensch, 1909 (Danainae), ambas simpátricas com T. h. caissara, porém bem mais comuns que esta.

\section{Hyalyris fiammetta (Hewitson, 1852) Ithomiinae, Nymphalidae}

Fig. 18

Situação no Brasil definida pelo IBAMA (Portaria 1522/89): provavelmente extinta.

Situação em Minas Gerais (deliberação COPAM 041-95): ameaçada, criticamente em perigo.

Distribuição em Minas Gerais: Conhecida de poucos lugares a leste do Estado na Floresta Atlântica, hoje a parte mais destruída, em encostas baixas e super-úmidas das serras altas. Data - Cachimbo, Peçanha - Cantagalo e Palma.

Ocorrência em outros Estados: Bahia, Espírito Santo (Alegre, Muqui), norte do Rio de Janeiro (Itabapoana) e São Paulo.

Hyalyris fiammetta (grafia correta) é uma borboleta média (asa anterior $30 \mathrm{~mm}$ ) de asas alongadas na qual o padrão mimético "tigre" de listras amarelas, alaranjadas e pretas está parcialmente dissolvido por uma difusão esbranquiçada. Isto reduz as áreas não pretas nas asas a uma mancha alaranjada basal, a uma faixa branca subapical e duas manchas brancas entre estas na asa anterior e a uma larga faixa branca no centro da asa posterior; todas as asas têm pontos brancos marginais e a face ventral é muito semelhante à dorsal. A fêmea retém um pouco mais de pigmento amarelo nas áreas brancas.

\section{Hyalyris leptalina (C. Felder \& R. Felder, 1865) Ithomiinae, Nymphalidae}

Fig. 19

Situação no Brasil definida pelo IBAMA (Portaria 1522/89): provavelmente extinta.

Situação em Minas Gerais (deliberação COPAM 041-95): ameaçada, em perigo. Distribuição em Minas Gerais: conhecida de poucos lugares da Floresta Atlântica, em serras baixas com encostas frias no leste e no sudeste, hoje todos destruidos; ainda ocorre no médio vale do Rio Doce. Teófilo Otoni, Cataguazes e Itueta.

Ocorrência em outros estados: Espírito Santo (Baixo Guandu, Itaguaçu, Mucurici) e Rio de Janeiro (Rio de Janeiro - Covanca de Jacarepaguá, Gávea, Saquarema - Lagoa de Saquarema).

Hyalyris leptalina é uma borboleta média (asa anterior 27-30mm), de asas alongadas e amarelas, com o terço apical da asa anterior branco, pouco transparente. Para o sul (no Rio de Janeiro e em Cataguazes - nenhuma população conhecida 
atualmente) a área branca é mais opaca e separada da amarela basal por uma faixa cuneiforme larga, preta e difusa; mais ao norte (Teófilo Otoni, Ituêta) a asa anterior é mais transparente e a marca preta mais fraca. Assim assemelha-se a diversos outros Ithomiinae simpátricos (Napeogenes yanetta (Hewitson, 1867), N. sulphurina Bates, 1862, Pteronymia euritea (Cramer, 1780), P. hemixanthe (C. Felder \& R. Felder, 1865), Scada karschina (Herbst, 1792), Hypoleria fallens (Haensch, 1905) e Epityches eupompe (Geyer, 1832)) (BROWN 1979, 1991, 1996a, b; MIELKE \& BROWN 1979).

\section{Hypoleria fallens (Haensch, 1905) \\ Ithomiinae, Nymphalidae}

Fig. 20

Situação no Brasil definida pelo IBAMA (Portaria 1522/89): ameaçada de extinção.

Situação em Minas Gerais (deliberação COPAM 041-95): ameaçada, vulnerável.

Distribuição em Minas Gerais: matas úmidas da Floresta Atlântica de até $300 \mathrm{~m}$ de altitude no vale do Rio Doce. Coronel Fabriciano, Marliéria, Raul Soares.

Ocorrência em outros Estados: Espírito Santo (Linhares) e Rio de Janeiro (Rio Dourado).

Hypoleria fallens é uma borboleta de tamanho médio (asa anterior $25 \mathrm{~mm}$ ), de asas alongadas, amarelas na metade basal, transparentes na metade apical, com uma mancha cuneiforme negra nas discocelulares, margens negras; a face ventral semelhante, porém as bordas com manchas difusas e a parte basal da margem costal alaranjada. É a única espécie do gênero com pigmento amarelo (3-hidroxi-quinurenina) armazenado nas asas.

\section{Arawacus aethesa (Hewitson, 1867) \\ Theclinae, Lycaenidae \\ Figs 21, 22}

Situação no Brasil definida pelo IBAMA (Portaria 1522/89): não consta da portaria.

Situação em Minas Gerais (deliberação COPAM 041-95): ameaçada, em perigo.

Distribuição em Minas Gerais: matas perenifolias ou semideciduas da Floresta Atlântica no vale do Rio Doce. Marliéria.

Ocorrência em outros Estados: Bahia e Espírito Santo (Conceição da Barra, Linhares).

Arawacus aethesa é uma borboleta pequena (asa anterior $13 \mathrm{~mm}$ ) com as asas de cor castanho-claro, na face ventral com margens e listras basais amarelas e alaranjadas, linhas negras convergentes no ângulo anal para uma "falsa cabeça" com "olhos" (pontos negros) e "antenas" (prolongamentos da veia CuA2 das asas posteriores), dando a impressão de que a cabeça está localizada na parte caudal; uma estratégia de defesa. 


\section{Cyanophrys bertha (Jones, 1912) \\ Theclinae, Lycaenidae}

Fig. 23

Situação no Brasil definida pelo IBAMA (Portaria 1522/89): não consta da portaria.

Situação em Minas Gerais (deliberação COPAM 041-95): ameaçada, vulnerável.

Distribuição em Minas Gerais: serra altas do sudeste, com matas úmidas e primárias da Floresta Atlântica. Barbacena e Poços de Caldas.

Ocorrência em outros Estados: Rio de Janeiro, São Paulo (Jundiaí), Paraná (Curitiba, Ponta Grossa, Castro), Santa Catarina.

Cyanophrys bertha é uma borboleta pequena (asa anterior $15 \mathrm{~mm}$ ) de coloração azul iridescente na face dorsal (mais clara e não iridescente na fêmea) e verde intenso na face ventral, nesta com faixa branco leitosa irregular, internamente acompanhada por desenhos violetas e brancos, entre o centro da margem costal e o ângulo anal, este vermelho, e com desenhos difusos vermelho escuro ao longo da margem externa; na face ventral da asa anterior com faixa subapical branca. A cauda da asa posterior, característica dos Theclinae e formada pelo prolongamento de $\mathrm{CuA} 2$, com 3-5mm e juntamente com a mancha vermelha do ângulo anal formam a "falsa cabeça", uma estratégia de defesa, como em Arawacus aethesa (BRown 1992, 1993, 1996a, b; CASAGRANDE \& MielKe 1993, 1995).

\section{Magnastigma julia Nicolay, 1977 \\ Theclinae, Lycaenidae \\ Fig. 24}

Situação no Brasil definida pelo IBAMA (Portaria 1522/89): não consta da portaria.

Situação em Minas Gerais (deliberação COPAM 041-95): ameaçada, vulnerável.

Distribuição em Minas Gerais: transição entre a Floresta Atlântica e o Cerrado. Barbacena.

Ocorrência em outros Estados: Distrito Federal (Brasília).

Magnastigma julia é uma borboleta muito pequena (asa anterior $9 \mathrm{~mm}$ ), marrom escura, com mancha androconial negra muito grande na margem costal da face dorsal e pequenos pontos vermelhos submarginais na face ventral da asa posterior; as franjas e caudas brancas. Representa a menor espécie e a menos colorida dentro do gênero; é quase invisível quando voa rapidamente entre hastes de plantas arbustivas ou gramíneas.

Nirodia belphegor Westwood, 1851

Riodininae, Riodinidae

Fig. 25

Situação no Brasil definida pelo IBAMA (Portaria 1522/89): não consta da portaria. 
Situação em Minas Gerais (deliberação COPAM 041-95): ameaçada, vulnerável.

Distribuição em Minas Gerais: nos campos rupestres de localidades isoladas na Serra do Espinhaço, em campos úmidos de altitude. Diamantina, Jabuticatubas (Serra do Cipó), Santa Bárbara (Caraça).

Ocorrência em outros Estados: desconhecida.

Nirodia belphegor é uma borboleta relativamente pequena (asa anterior $20 \mathrm{~mm}$ ), de asa pontuda e com um prolongamento caudal na asa posterior curto e largo, na face dorsal com azul iridescente escuro, cobrindo grande parte das asas, e com duas a quatro manchas brancas subapicais no limite do azul com o ápice negro; na asa posterior com uma mancha amarelo-rosada no ápice e outra vermelha no ângulo anal, franjas entre as veias brancas; a face ventral é de um castanho escuro e as manchas da face dorsal se repetem, e ainda há mais uma vermelha na margem interna e duas discais na proximidade da cauda.

\section{REFERÊNCIAS BIBLIOGRÁFICAS}

BERNARDES, A.T.; A.B. MACHADO \& A.B. RYLANDS. 1990. Fauna brasileira ameaçada de extinção. Belo Horizonte, Fundação Biodiversitas, 65p.

BROWN JR., K.S. 1970. Rediscovery of Heliconius nattereri in eastern Brazil. Ent. News 81: 129-140.

1972a. Maximizing daily butterfly counts. Jour. Lep. Soc. 26: 183-196. 1972b. The Heliconians of Brazil (Lepidoptera: Nymphalidae). Part III. Ecology and biology of Heliconius nattereri, a key primitive species near extinction, and comments on the evolutionary development of Heliconius and Eueides. Zoologica 57: 41-69.

. 1979. Ecologia geográfica e evolução nas florestas neotropicais. Campinas, Unicamp, XXXI+265+120p.

1991. Conservation of neotropical environments: insects as indicators, p.349-404. In: N.M. Collins \& J.A. ThOMAS (Eds). The conservation of insects and their habitats. London, Academic Press.

1992. Borboletas da Serra do Japi: Diversidade, habitats, recursos alimentares e variação temporal, p. 142-187. In: L.P.C. Morellato (Ed.). História natural da Serra do Japi: Ecologia e preservação de uma área florestal no sudeste do Brasil. Campinas, Unicamp/ Fapesp.

. 1993. Neotropical Lycaenidae: an overview, p.45-61; Selected Neotropical species, p. 146-149; Theclinae endemic to the Cerrado vegetation (central Brazil), p.152. In: T.R. NEW (Ed.). Conservation biology of Lycaenidae (Butterflies). Gland, IUCN.

1996a. Conservation of threatened species of Brazilian butterflies, p.45-62. In: S.A. AE; T. HIROWATARI; M. ISHII \& L. P. BRoWER (Eds.). Decline and conservation of Butterflies in Japan III. Osaka: Lepid. Soc. Japan; Yadoriga, special issue.

1996b. Diversity of Brazilian Lepidoptera: History of study, methods for measurements, and use as indicator for genetic, specific and system richness, p.121-153. In: C.E.M. BICUDO \& N.A. MENEZES (Eds). Biodiversity in Brazil: a first approach. São Paulo, Instituto de Botânica/CNPq. 
Brown, JR., K. S. \& O.H.H. MielKe. 1967a. Lepidoptera of the central Brazil plateau. I. Preliminary list of Rhopalocera: Introduction, Nymphalidae, Libytheidae. Jour. Lep. Soc. 21: 77-106

1967b. Lepidoptera of the central Brazil plateau. II. Preliminary list of Rhopalocera: Lycaenidae, Pieridae, Papilionidae, Hesperiidae. Jour. Lep. Soc. 21: $145-168$.

1968. Lepidoptera of the central Brazil plateau. III. Partial list for the Belo Horizonte area, showing the character of the southeastern "blend zone". Jour. Lep. Soc. 22: 147-157.

ButLER, A.G. 1869. Descriptions of new Rhopalocera from the collection of Herbert Druce. Cist. Ent. 1, p. 1-16.

Casagrande, M. M. \& O. H. MielKe. 1993. Borboletas (Lepidoptera) ameaçadas de extinção no Paraná. Revta bras. Zool. 9: 75-92.

—. 1995. Borboletas ameaçadas de extinção no Paraná, p.143-157. In: Paraná, Sema. Lista vermelha dos animais ameaçados de extinção no Estado do Paraná. Curitiba, Sema/GTZ.

EBERT, H. 1969. On the frequency of butterflies in eastern Brazil, with a list of the butterfly fauna of Poços de Caldas, Minas Gerais. Jour. Lep. Soc. 23 (Suppl. 3): $1-48$.

Foetterle, J.G. 1902. Descripção de Lepidopteros novos do Brazil. Revta Mus. Paulista 5: 616-652.

FortunAto, L. \& A. RuszCZYK. 1997. Comunidades de lepidópteros frugívoros em áreas verdes urbanas e extraurbanas em Uberlândia, MG. Rev. Brasil. Biol. 57: 79-87.

HAMBLETON, E.J. \& W. T.M. ForBES. 1935. Uma lista de Lepidoptera (Heterocera) do Estado de Minas Gerais. Arch. Inst. Biol., São Paulo, 6: 213-256.

HEPPNER, J.B. 1991. Faunal regions and the diversity of Lepidoptera. Trop. Lep. 2 (Suppl. 1): 1-85.

HigGins, L.G. 1981. A revision of Phyciodes Hübner and related genera, with a review of the classification of the Melitaeini (Lepidoptera: Nymphalidae). Bull. Brit. Mus. (Nat. Hist.), Ent. 43: 77-243.

Kesselring, J. 1989. Agrias, a rainha das borboletas. Ciencia Hoje, Rio de Janeiro, 10: $40-48$.

MielKe, O.H. \& K. S. BROWN JR. 1979. Suplemento ao Catálogo dos Ithomiidae americanos (Lepidoptera) de Romualdo Ferreira D'Almeida (Nymphalidae: Ithomiinae). Curitiba, UFPR/CNPq, VII+216p.

MinAS GERAIS 20/ I/1996. Deliberação COPAM no 041/95. Aprova a lista de espécies ameaçadas de extinção da fauna do Estado de Minas Gerais. Minas Gerais. Órgão Oficial dos Poderes do Estado. Diário Executivo, Legislativo e Publicações de Terceiros, Belo Horizonte, 104 (14), parte I, p.1-4.

TYLER, H.A.; K.S. BROWN JR. \& K.H. WILSON. 1994. Swallowtail butterflies of the Americas. A study in biological dynamics, ecological diversity, biosystematics, and conservation. Gainesville, Florida, Scientific Publishers, 376p. ZIKÁN, J.F. \& W. ZIKÁN. 1968. Inseto-fauna do Itatiaia e da Mantiqueira. III. Lepidoptera. Pesq. agropec. bras. 3: 45-109.

Recebido em 28.II.1997; aceito em 06.V.1998.

Revta bras. Zool. 15 (1): 241 - 259, 1998 